\title{
Detecçáo precoce do câncer de mama na mídia brasileira no Outubro Rosa
}

\author{
| ${ }^{1}$ Mônica De Assis, ${ }^{2}$ Renata Oliveira Maciel dos Santos, ${ }^{3}$ Arn Migowski |
}

Resumo: O estudo objetivou analisar como a informação sobre detecção precoce do câncer de mama é apresentada na mídia brasileira durante o Outubro Rosa. Foram analisadas matérias jornalísticas publicadas na internet em outubro de 2017. A busca recuperou 1.011 matérias, que, organizadas e selecionadas, resultaram em 345 elegíveis. Os principais porta-vozes foram os mastologistas $(17,1 \%)$ e os pacientes $(13,0 \%)$, e os vínculos institucionais predominantes foram público $(36 \%)$ e privado (34\%). A detecção precoce foi o segundo tema mais frequente (55\%), e o rastreamento mamográfico foi tratado em 79,1\% dessas matérias. Predominou a recomendação de iniciar o rastreamento mamográfico na faixa etária de 40 anos $(34,3 \%)$ comparada à de 50 anos (29,3\%). A recomendação governamental de rastreamento bienal de 50 a 69 anos foi citada em 17,5\% das matérias. Apenas 3,3\% citaram os riscos do rastreamento e $1,5 \%$, a importância da decisão compartilhada. Os sinais e sintomas suspeitos de câncer de mama foram divulgados em 30\% das matérias, e apenas $11,8 \%$ listaram todas as principais alteraçóes. A carência de informaçóes e a presença de recomendaçóes inadequadas ou incompletas a respeito de faixa etária e periodicidade mostram o limite da campanha em disseminar informaçôes baseadas nas melhores evidências.

> Palavras-chave: neoplasias da mama; detecção precoce de câncer; programas de rastreamento; mamografia; comunicação em saúde.

\author{
${ }^{1}$ Divisão de Detecção Precoce e \\ Apoio à Organização de Rede, \\ Instituto Nacional do Câncer. Rio \\ de Janeiro-RJ, Brasil (monassis. \\ mm@gmail.com). \\ ORCID: 0000-0002-7137-9471 \\ ${ }^{2}$ Divisão de Detecção Precoce e \\ Apoio à Organização de Rede, \\ Instituto Nacional do Câncer. Rio \\ de Janeiro-RJ, Brasil (renamsant@ \\ yahoo.com.br). \\ ORCID: 0000-0002-6747-0184 \\ ${ }^{3}$ Divisão de Detecção Precoce e \\ Apoio à Organização de Rede, \\ Instituto Nacional do Câncer. Rio \\ de Janeiro-RJ, Brasil (arn.santos@ \\ inca.gov.br). \\ ORCID: 0000-0002-4861-2319
}

Recebido em: 29/03/2019 Aprovado em: 16/12/2019 Revisado em: 23/03/2020 


\section{Introdução}

Desde sua introduçâo no Brasil, no início dos anos 2000, o movimento Outubro Rosa vem ganhando adesão da sociedade e é hoje uma das campanhas mais populares da área da saúde. Pacientes, serviços de saúde, empresas e organizaçóes da sociedade civil vêm se engajando na causa do câncer de mama e protagonizando açôes de mobilização social sobre a doença.

O câncer de mama é o que mais acomete as mulheres em todo o mundo e também no Brasil, depois do câncer de pele não melanoma. Em 2020, são esperados 66.280 novos casos, o que representa $27 \%$ dos casos de câncer em mulheres (INCA, 2019). A mortalidade por essa neoplasia é ainda ascendente no país, sendo registrados, em 2018, 17.572 óbitos por câncer de mama em mulheres (INCA, 2020). Avanços terapêuticos nas últimas décadas vêm ampliando as possibilidades de enfrentamento da doença, porém a detecção precoce segue como desafio.

A detecção precoce do câncer de mama visa a identificar a doença em fase inicial, seja por meio do diagnóstico precoce, estratégia dirigida às mulheres com sinais e sintomas suspeitos da doença, ou do rastreamento mamográfico, exames de rotina em mulheres assintomáticas em faixa etária e periodicidade definidas.

A mamografia é considerada o exame padrão para rastrear o câncer de mama, mas tem limites e riscos que precisam ser conhecidos, tais como: resultados falsos positivos, que causam ansiedade e necessidade de mais exames; resultados falsos negativos, que causam falsa segurança para a mulher; excesso de diagnóstico e de tratamento (sobrediagnóstico e sobretratamento), quando tumores que podem não evoluir são identificados e tratados; e ainda o pequeno risco de exposição à radiação ionizante, que pode aumentar com repetiçóes de mamografias ao longo de muitos anos (MIGOWSKI et al., 2018a; INCA, 2015).

Considerando a necessidade de se buscar o balanço favorável entre riscos e benefícios do rastreamento, a recomendação do Ministério da Saúde é que a mamografia de rastreamento seja ofertada para mulheres de 50 a 69 anos, a cada dois anos (INCA, 2015). As mulheres devem ser informadas sobre os riscos e benefícios envolvidos para que possam avaliar participar ou não do rastreio, em um processo de decisão compartilhada (MIGOWSKI et al., 2018b). As diretrizes brasileiras foram baseadas em revisões sistemáticas da literatura (MIGOWSKI et al., 2018c) e suas recomendações de população-alvo e periodicidade estão em consonância com as da 
Organização Mundial da Saúde (OMS) e da maioria dos países que implantaram o rastreamento populacional do câncer de mama (WHO, 2014; EBELL et al., 2018).

Há, porém, outra recomendação para o rastreamento mamográfico no Brasil. Nela, sociedades de especialidades médicas recomendam a mamografia anual para mulheres de 40 a 74 anos (), em uma diretriz com menor rigor metodológico (URBAN et al., 2017; MIGOWSKI et al., 2018c). Embora essa diretriz não considere explicitamente os riscos mais importantes do rastreamento, é possível encontrar, de forma periférica, a menção à necessidade de orientar as mulheres quanto aos riscos e benefícios do rastreamento, em um posicionamento, em nível regional, de uma das sociedades envolvidas (VIEIRA, 2017).

A mídia possui papel fundamental na formação da opinião pública e na difusão de informações de saúde e de recomendações para a população. Entretanto, nem sempre os conteúdos veiculados possuem qualidade adequada e refletem, muitas vezes, interesses privados (EMERICH et al., 2016). Esse fato, associado à tendência contemporânea de consumo excessivo e acrítico de tecnologias em saúde (VASCONCELLOS-SILVA et al., 2010), perpetua práticas que podem ser potencialmente danosas, como é o caso do rastreio mamográfico em faixas etárias e periodicidades não recomendadas.

O papel da mídia na comunicação sobre o câncer de mama vem sendo problematizado em estudos em nível internacional (ABIGHANNAM; CHILEK; $\mathrm{KOH}$, 2018) e nacional (SIMEÃO et al., 2016; VASCONCELLOS-SILVA; SORMUNEN; CRAFTMAN, 2018). Porém, a temática específica que envolve a detecção precoce, e especialmente as questôes contemporâneas sobre o rastreamento mamográfico, são ainda pouco estudadas. Buscou-se, então, responder aqui à pergunta: como a informação sobre detecção precoce do câncer de mama é apresentada na mídia brasileira?

Tendo em vista a grande atenção dada pela mídia a essa questão no mês de outubro e a existência de múltiplas vozes quanto às recomendaçôes para a população, o objetivo deste artigo é analisar como a informação sobre a detecção precoce do câncer de mama é apresentada na mídia brasileira no contexto do Outubro Rosa.

\section{Metodologia}

Foi realizada análise documental das matérias jornalísticas da base de dados da XClipping - Monitoramento Estratégico da Mídia, empresa privada que provê 
diariamente uma lista de matérias sobre câncer, publicadas na mídia brasileira, para uma instituição especializada em câncer. Essa base, segundo estimativa da empresa, abrange em torno de $90 \%$ da mídia nacional e subdivide-se em: impressos, internet, rádio, televisão e redes sociais.

A busca de matérias foi feita com o filtro "internet" e a palavra-chave "câncer de mama", no período de 01 a 31 de outubro de 2017. A base de "internet" é a que tem maior volume, pois inclui também parte do conteúdo da base de "impressos" cujos veículos possuam canal digital. A busca resultou em 1.011 matérias.

Foi realizada leitura do título e conteúdo para exclusão de matérias repetidas ou muito similares publicadas em veículos diferentes. Observou-se que é comum a replicação de notícias pelos veículos de menor porte, o que resultou na eliminação de 445 matérias. Nessa etapa, foram também excluídas seis matérias por não abordarem o câncer de mama.

A segunda etapa foi a leitura completa das 560 matérias restantes para identificação das elegíveis para análise. Considerou-se como critério de inclusão a presença na matéria de conteúdos relacionados a um ou mais dos eixos temáticos de interesse desta pesquisa: rastreamento, diagnóstico precoce e prevenção primária. A inclusão do tema prevenção primária se deu por ser essa uma estratégia fundamental para o controle do câncer de mama, assim como a detecção precoce, no cenário de enfrentamento das doenças crônicas não transmissíveis (BRASIL, 2011). Foram consideradas elegíveis 345 matérias que abordaram deteç̧ão precoce ou prevenção primária (figura 1).

Para a coleta de dados, utilizou-se um instrumento elaborado no Google Forms, que possibilita o preenchimento simultâneo por dois pesquisadores, com armazenamento automático dos dados em planilha formato Excel. O formulário foi composto por questôes predominantemente fechadas, com campos abertos para observaçóes. Foram incluídas as seguintes variáveis: Porta-voz: perfil e vínculo institucional; Faixa etária e periodicidade do rastreamento do câncer de mama; Riscos e benefícios da mamografia de rastreamento; Decisão informada sobre rastreamento; Atenção da mulher às suas mamas (breast awareness); Técnica e/ou rotina para fazer autoexame das mamas; Principais alteraçôes suspeitas de câncer de mama, tais como: nódulo; endurecimento de partes da mama; mudança na pele; saída espontânea de líquido do mamilo; vermelhidão; mudança na posição ou formato do mamilo; nódulo no pescoço ou axilas; e Prevenção primária. 
O instrumento de coleta de dados foi submetido a um pré-teste por meio da análise de 30 matérias, analisadas separadamente por duas pesquisadoras. Adequações foram feitas para garantir maior padronização. Para maior uniformidade na coleta, foi elaborado um manual com os critérios para responder a cada variável do formulário.

A sistematização dos dados foi feita no programa Excel, utilizando-se estatísticas descritivas simples para análise das questôes fechadas. Os dados qualitativos foram sistematizados e categorizados, seguindo princípios da análise de conteúdo com préanálise, exploração do material e tratamento dos resultados (BARDIN, 2011).

Figura 1. Fluxograma da busca e coleta de dados

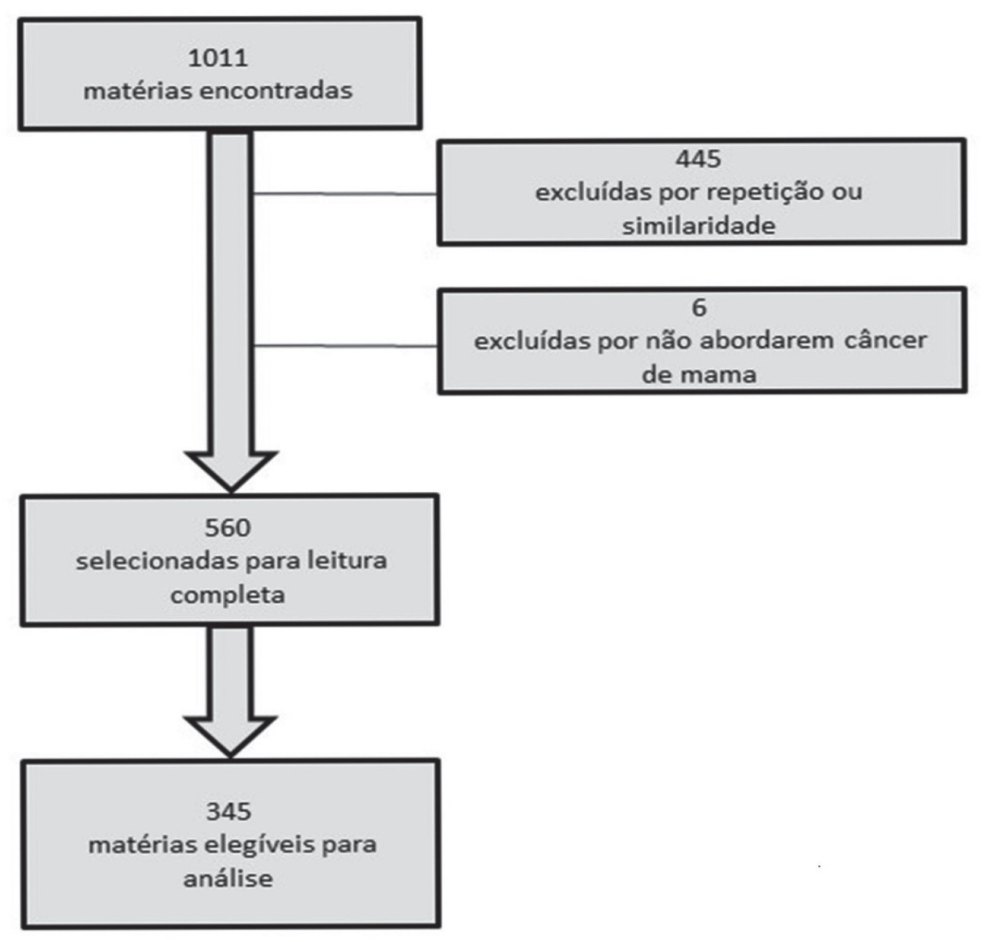

\section{Resultados}

A tabela 1 mostra um panorama dos assuntos abordados no conjunto total das matérias, apresentadas por 626 veículos, incluindo os de grande circulação como $O$ Globo, Estadão, Correio Popular, Portal G1, Zero Hora, Veja, Band, dentre outros. Em 
geral, há mais de um assunto por matéria, sendo os mais recorrentes o próprio Outubro Rosa e a divulgação de açôes de mobilização e apoio social (palestras, debates, oferta de serviços, caminhadas/práticas esportivas, exposições, ações solidárias, dentre outras) (91,3\%). A detecção precoce foi o segundo tema mais frequente (55,3\%), seguido dos dados epidemiológicos sobre a magnitude do câncer de mama, produção dos serviços e outros (49,8\%). Prevenção primária $(24,8 \%)$ e fatores de risco $(18,7 \%)$ apareceram também dentre os principais temas abordados. Muitas matérias ilustraram os assuntos com histórias de mulheres que enfrentaram ou estão enfrentando a doença. A oferta de mamografia e de outros serviços, bem como o déficit nessa área, também foi destacada, dentre outros diversos e variados temas.

Tabela 1. Temas abordados nas matérias sobre câncer de mama publicadas no mês de outubro de 2017

\begin{tabular}{|l|c|c|}
\hline Temas & No & $\%$ \\
\hline Outubro rosa / ações de mobilização / ações solidárias & 515 & 91,3 \\
\hline Deteç̧ão precoce & 312 & 55,3 \\
\hline Incidência / mortalidade / outros aspectos epidemiológicos & 281 & 49,8 \\
\hline Prevenção primária & 140 & 24,8 \\
\hline Fatores de risco & 94 & 16,7 \\
\hline Oferta ou deficit de serviços & 86 & 15,2 \\
\hline Tratamento & 65 & 11,5 \\
\hline Vivência de pacientes & 53 & 9,4 \\
\hline Reconstrução mamária & 28 & 5 \\
\hline Tecnologias diagnósticas & 21 & 3,7 \\
\hline Divulgação institucional & 21 & 3,7 \\
\hline Aspectos genéticos & 13 & 2,3 \\
\hline Direitos das pacientes & 11 & 2 \\
\hline Outros & 32 & 5,7 \\
\hline
\end{tabular}

Fonte: elaboração própria.

Foram identificados 315 porta-vozes no total, em geral mais de um por matéria, que utilizaram esse recurso. Os principais foram os mastologistas e os pacientes, 
seguidos pelos oncologistas e outros profissionais de saúde não médicos ou médicos generalistas. Autoridades sanitárias, tais como ministro, secretários e representantes de áreas técnicas, nos vários níveis, também foram presentes, além de políticos e profissionais de empresa privadas, das áreas de gestão, recursos humanos e marketing. Representantes de organizações não governamentais (ONGs) foram porta-vozes em 20 matérias (gráfico 1).

Os vínculos institucionais dos porta-vozes foram o sistema público de saúde (36\%), o sistema privado de saúde (34\%) e as ONGs (18\%). Pacientes sem vínculo institucional foram também porta-vozes (13\%) e, em $16,3 \%$ dos casos, não foi possível identificar o vínculo.

Gráfico 1. Porta-vozes identificados nas matérias sobre câncer de mama publicadas no mês de outubro de 2017

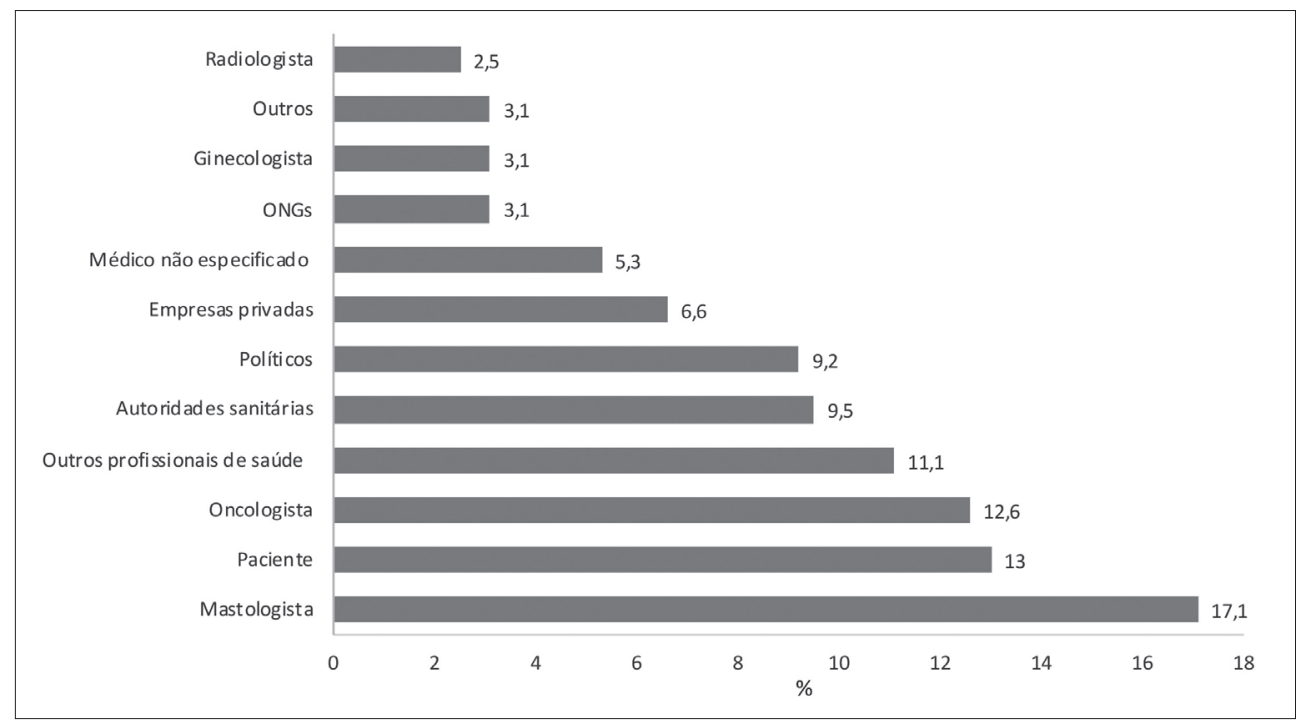

Fonte: elaboração própria.

O rastreamento - exames periódicos em população assintomática - foi tratado em 273 matérias que abordaram o tema detecção precoce $(79,1 \%)$. As recomendaçóes para a mamografia de rastreamento foram as mais diversas e, em alguns casos, mais de uma foi mencionada na mesma matéria, resultando em 297 registros.

Desse total, 31,3\% das matérias, apesar de divulgarem a mamografia, não fizeram nenhuma referência à periodicidade ou ao público-alvo do exame. A recomendação 
do Ministério da Saúde/INCA para o rastreio mamográfico (50 a 69 anos, a cada dois anos) foi citada em apenas $17,5 \%$ das matérias, frequência igual à da recomendação das sociedades médicas (a partir de 40 anos, anual). Quando agrupadas as variaçóes em torno dessas duas principais, predominou a recomendação com início na faixa etária de 40 anos (34,3\%) comparada à de 50 anos (29,3\%). Um pequeno número de matérias apontou a necessidade de mamografia antes dos 40 anos (3,7\%). Outras matérias se referiram à recomendação de mamografia aos 30 ou 35 anos; à continuidade anual desse exame a partir de 70 anos; e a diferentes proposições de periodicidade sem relacioná-las à faixa etária (tabela 2). A recomendação de periodicidade anual teve uma leve predominância com relação à periodicidade bienal (61 vs. 59).

Tabela 2. Recomendação de faixa etária e periodicidade da mamografia de rastreamento em mulheres com risco padrão para câncer de mama

\begin{tabular}{|l|c|c|}
\hline Recomendaçóes & No & $\%$ \\
\hline Náo especificou faixa etária e nem periodicidade & 93 & 31,3 \\
\hline Faixa etária 50 anos e mais & 52 & 17,5 \\
50 a 69 anos bienal & 17 & 5,7 \\
50 a 69 anos, sem mencionar a periodicidade & 11 & 3,7 \\
A partir dos 50 anos, sem mencionar periodicidade & 3 & 1,0 \\
A partir dos 50 anos, anual & 2 & 0,7 \\
A partir dos 50 anos, bienal & 2 & 0,7 \\
50 a 69 anos anual & 87 & 29,3 \\
Subtotal & & \\
Faixa etária 40 anos e mais & 52 & 17,5 \\
A partir de 40 anos, anual & 34 & 11,4 \\
A partir dos 40 anos, sem mencionar periodicidade & 8 & 2,7 \\
40 a 69 anos, sem mencionar periodicidade & 3 & 1,0 \\
40 a 69 anos anual & 3 & 1,0 \\
40 a 69 anos bienal & 2 & 0,7 \\
A partir de 40 anos bienal & & 34,3 \\
Subtotal & 32 \\
\hline
\end{tabular}




\begin{tabular}{|l|c|c|}
\hline Recomendaçóes & No & $\%$ \\
\hline Faixa etária abaixo de 40 anos & & \\
$\quad$ Aos 35 anos como base & 2 & 0,7 \\
A partir dos 30 anos, sem mencionar periodicidade & 1 & 0,3 \\
$\quad$ A partir dos 35 anos, anualmente & 1 & 0,3 \\
$\quad$ Subtotal & 4 & 1,3 \\
\hline Outras & 11 & 3,7 \\
\hline Total & 297 & 100 \\
\hline
\end{tabular}

Fonte: elaboração própria.

Somente nove matérias $(3,3 \%)$ abordaram que há recomendaçôes conflitantes para o rastreamento mamográfico no Brasil. Os riscos do rastreamento foram apontados nessas mesmas matérias, da seguinte forma: sete matérias referiramse ao sobrediagnóstico e ao sobretratamento; seis apontaram os exames invasivos desnecessários; cinco, os resultados falsos positivos; quatro, a exposição à radiação; e três, os resultados falsos negativos. Das matérias que abordaram os riscos, apenas quatro mencionaram a importância da decisão compartilhada, o que representou $1,5 \%$ do total de matérias que abordaram o rastreamento.

Dentre as matérias que apresentaram recomendação de rastreamento e nas quais o vínculo do porta-voz era privado, $74 \%$ recomendaram a realização da mamografia a partir dos 40 anos. Quando o vínculo do porta-voz era público, 62\% recomendaram a realização da mamografia a partir dos 50 anos.

A mensagem para a mulher ficar atenta às alteraçóes da mama apareceu em $63,4 \%$ das matérias, usando os termos autoexame, autoconhecimento ou afins. Muitas matérias apenas mencionaram a necessidade de autoexame, sem informação adicional. A orientação quanto ao uso de técnica ou periodicidade específica para o autoexame foi observada em $15,1 \%$ das matérias que mencionaram a importância de a mulher olhar as suas mamas, com diferentes mensagens: uma vez por mês, três a cinco dias após a menstruação ou em uma data fixa em mulheres na menopausa. Algumas matérias apresentaram limites do autoexame como método de detecção precoce, dando destaque à consulta médica e a métodos de imagem.

Apenas 30\% das matérias apresentaram sinais e sintomas suspeitos de câncer de mama, e somente 11,8\% citaram todas as alteraçôes suspeitas relevantes (gráfico 2). 
Gráfico 2. Apresentação das alterações suspeitas de câncer de mama nas matérias analisadas

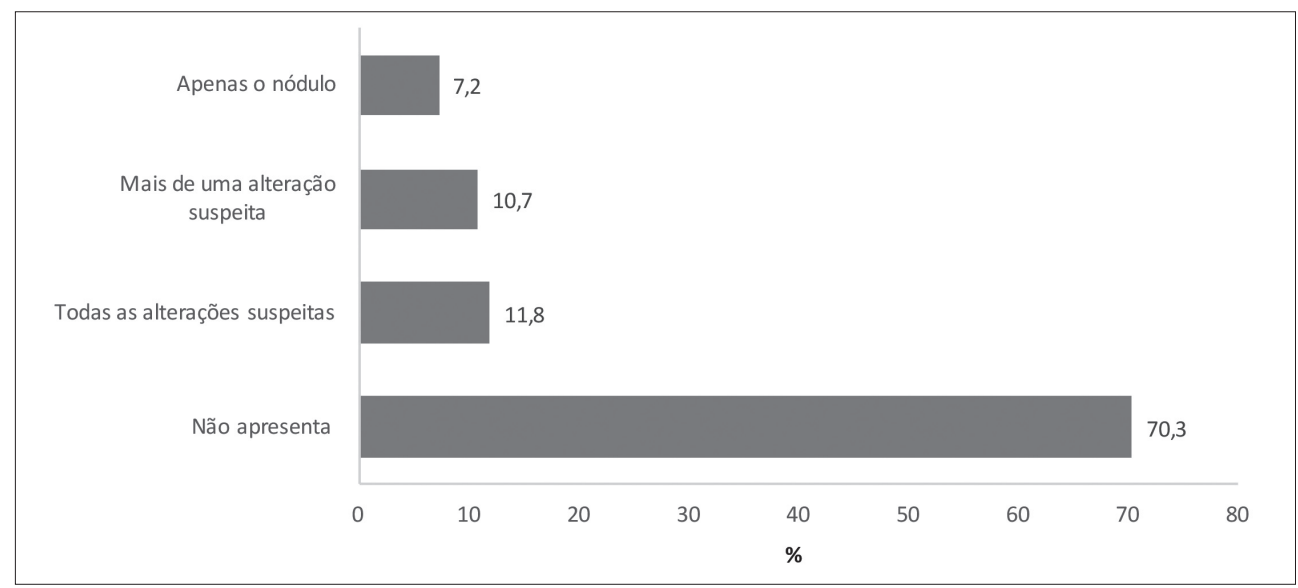

Fonte: elaboração própria.

A prevenção primária do câncer de mama, observada na informação sobre fatores modificáveis que reduzem o risco da doença, foi abordada em $42,4 \%$ das matérias. Os aspectos mais destacados foram a prática de atividade física e a alimentação saudável.

\section{Discussão}

O grande volume de matérias sobre câncer de mama no período analisado mostra a força do Outubro Rosa na sociedade brasileira e o interesse da mídia em relação ao tema, confirmando ser esse um momento de grande mobilização e disseminação de informações sobre a doença. Ações diversas de informação, apoio social e outros serviços são oferecidos às mulheres em geral e às que enfrentam o câncer de mama. Marcas institucionais, públicas e especialmente privadas, são divulgadas sob a lógica do marketing rosa.

Em que pese o inestimável valor do apoio social e da autoajuda para favorecer a autoestima e empoderar as mulheres que vivem a doença, é necessário um olhar crítico sobre os efeitos mercadológicos do marketing rosa, que podem secundarizar interesses da saúde pública e incorrer em questôes éticas (CARDOSO-FILHO; SANTOS; SHINZATO, 2015). É o caso, por exemplo, da promoção de produtos com algum potencial cancerígeno, das doaçóes pouco transparentes anunciadas da 
venda de produtos "rosa" e da própria secundarização de informaçôes de qualidade em um momento pretensamente informativo (ABIGHANNAM; CHILEK; KOH, 2017). O documentário norte-americano Pink Ribbon Inc, lançado em 2011, abordou esses dentre outros aspectos críticos do movimento Outubro Rosa no contexto dos Estados Unidos, onde nasceu e se disseminou internacionalmente (PINK, 2011). Para o escopo da presente pesquisa, foi possível constatar falhas na informação para o público, seja por lacunas ou equívocos.

Dentre os principais porta-vozes médicos, destacaram-se os mastologistas e oncologistas, confirmando seu papel de líderes de opinião sobre o tema, embora esses, geralmente, não tenham envolvimento direto com o rastreamento, seja na solicitação do rastreio, comumente feita por médicos de família, ginecologistas, clínicos gerais, geriatras ou enfermeiros, seja na leitura dos exames, papel dos radiologistas.

As duas principais recomendaçóes de faixa etária e periodicidade do rastreamento do câncer de mama no Brasil, vocalizadas por órgão governamental, de um lado, e sociedades de especialistas, de outro, foram igualmente presentes nas matérias jornalísticas, mas variaçóes incompletas e/ou incorretas foram também muito veiculadas. Raras matérias trouxeram ambas as recomendaçóes e fizeram contrapontos entre elas, informando sobre a controvérsia envolvida. De outra parte, a divulgação da mamografia de rastreamento sem informação sobre faixa etária e periodicidade, observada em mais de $30 \%$ das matérias, e a falta de informação sobre a periodicidade desse exame em muitas outras contribuem para fortalecer no imaginário da população a lógica do check-up médico como algo sempre positivo, não importando evidências sobre a segurança, benefícios e critérios para a sua utilização (MARTINS, 2005).

A recomendação frequente de se iniciar o rastreamento na faixa dos 40 anos ocorreu mesmo com a existência de evidências sobre importante aumento de riscos com essa prática e de provável ausência de benefícios, não havendo evidências conclusivas de redução da mortalidade por câncer de mama e nem mesmo de mudança de estadiamento (NELSON et al., 2016; MIGOWSKI et al., 2018a). Diversos fatores explicam esses resultados, entre eles a dificuldade à maior difusão de diretrizes que preconizam essa prática, a influência de líderes de opinião, dificuldades de compreender as evidências, conflito de interesses e prática de medicina defensiva (GIGERENZER, 2002.; MIGOWSKI et al., 2018b). 
Os riscos do rastreamento do câncer de mama foram praticamente ausentes nas matérias - apenas nove abordaram esse tema -, o que é uma lacuna de informação prejudicial tanto para a mulher quanto para o sistema de saúde, em função do sobrerrastreamento e do sobrediagnóstico e dos riscos associados a exames e tratamentos desnecessários. Estudo recente em municípios de Minas Gerais encontrou $21 \%$ de sobrerrastreio por excesso de periodicidade na mamografia de rastreamento, valor que aumentou para $24 \%$ durante as campanhas do Outubro Rosa (RODRIGUES et al., 2019).

A discussão sobre riscos e benefícios das intervençôes é u ma das mais importantes premissas em saúde. Toda intervenção populacional deve ter sua segurança e efetividade comprovadas, cumprindo o que se propóe e causando o menor dano possível (BRASIL, 2010). A estratégia para lidar com o risco de sobrediagnóstico e tratamento excessivo é adotar a decisão individualizada e compartilhada, considerando os potenciais benefícios e os conhecidos danos (LOWENSTEIN et al., 2018). A decisão compartilhada é um processo colaborativo entre profissional de saúde e usuário, que inclui partilhar informaçôes e considerar preferências e valores, garantindo que essas sejam incluídas no plano de cuidado (ELWYN et al., 2017). Essa perspectiva foi praticamente ausente nas matérias jornalísticas - apenas quatro a mencionaram -, o que confirma a total incipiência desse debate no Brasil (ABREU et al., 2011).

Nesse aspecto é grande a distância do Brasil em relação a países que incorporaram a decisão compartilhada no rastreamento organizado do câncer de mama. Materiais educativos de vários países contemplam essa questão, inclusive apresentando números para ilustrar em que proporção os riscos e benefícios do rastreamento ocorrem numa dada realidade (GOUVERNEMENT DU QUÉBEC, 2016; AUSTRALIAN GOVERNMENT, s/d; NHS, 2018). Esses materiais atuam como ferramentas de apoio à decisão, auxiliando as mulheres a realizarem escolhas mais adequadas aos seus valores e preferências (MERCHANT; DICKERT; HOWARD, 2018).

No Brasil há iniciativas voltadas à decisão das mulheres em temas como esterilização e via do parto, algumas doenças crônicas e avanços da participação dos usuários nas decisões sobre saúde, após a Política de Humanização. É consenso, porém, que a decisão compartilhada é ainda pouco implementada, ficando a cargo de cada profissional, que, na maioria das vezes, não considera as preferências individuais para a tomada de decisão (ANDREZZO, 2016; ROCHA et al., 2017; YAMAMOTO, 
2017; ABREU et al., 2006, 2011; STEPHAN et al., 2018). Não foram identificados materiais nacionais de apoio à decisão no rastreamento do câncer de mama.

O fato de essa discussão ser quase ausente na mídia brasileira indica, muito provavelmente, que ela não está presente em grande parte das práticas assistenciais do sistema público e do privado, uma vez que representantes de ambos figuraram igualmente como porta-vozes nas matérias com um pouco mais de $30 \%$ cada. Por outro lado, interesses corporativos e mercadológicos também podem contribuir para essa omissão, já que, em torno da mamografia e de outros exames diagnósticos, um grande mercado é movimentado, o que exige fortalecer o movimento em prol da prevenção quaternária que cresce no Brasil, em anos recentes (SILVA, 2014).

A informação para a mulher ficar atenta às suas mamas figurou entre as mensagens centrais do Outubro Rosa, embora com menor força que a mensagem sobre a mamografia $(62 \%$ contra $79,1 \%)$. A referência genérica ao autoexame foi mais frequente do que a perspectiva da estratégia de conscientização (breast awareness), o que mostrou ser essa uma visão menos comum no contexto brasileiro.

A circulação de mensagens sobre técnica e/ou rotina para fazer autoexame das mamas, no sentido clássico do termo, foi, por sua vez, pequena, demonstrando que o termo científico de autoexame como método padronizado comumente não corresponde ao significado desse termo para a mídia leiga e a população em geral. O ideal é que as mensagens sobre técnicas e periodicidade de autoexame sejam atualizadas para a visão que propóe o reconhecimento e a atenção com as mamas em situaçóes da vida cotidiana que sejam adequadas e confortáveis para a mulher. Destaca-se que o ensino do método padronizado de autoexame não é mais oficialmente recomendado no país há 19 anos (MIGOWSKI et al., 2018c). $\mathrm{Na}$ linha do breast awareness, a mulher deve reconhecer o que é o padrão normal para as suas mamas e o que são alteraçôes suspeitas, a fim de buscar rapidamente o serviço de saúde para investigação diagnóstica (MAcCREADY; LITTLEWOOD; JENKINSON, 2005). A mídia poderia contribuir muito para a difusão dessa mensagem, porém apenas $11,8 \%$ das matérias informaram sobre todas as alteraçôes suspeitas, valor que subiu para cerca de $30 \%$ quando a informação foi parcial. Essa informação - junto com a compreensão de que o câncer de mama, mesmo quando detectado por meio de sinais e sintomas, tem tratamento e que vale identificá-lo em fase inicial - é elemento-chave para a redução do estadiamento da doença no momento do diagnóstico. 
A prevenção primária do câncer de mama foi um tema presente em pouco menos da metade do conjunto de matérias elegíveis, porém de forma mais frequente do que o observado no estudo de Simeão et al. (2016), que analisou dados da mídia impressa sobre o tema, nos anos 2012 e 2013, e concluiu que o sentido de "prevençáo" dado ao tema focava predominantemente na realização de exames de detecção precoce (prevenção secundária), pouco abordando as práticas comportamentais para redução do risco da doença. Por certo, essa náo deixa de ser ainda uma tônica da campanha, mas a visibilidade do assunto vem crescendo, demonstrando refletir as evidências científicas que, nos últimos anos, vêm reforçando a margem importante de redução do risco da doença, por fatores modificáveis (WCRF; AICR, 2018).

Um aspecto interessante observado nas matérias, talvez para romper alguns limites de uma campanha focada numa só doença, foram as propostas de articulação de ações sobre câncer de mama com outras temáticas de saúde da mulher. Esse formato foi o adotado principalmente por secretarias de Saúde, que aproveitaram o momento para impulsionar o controle do câncer do colo do útero e outras açóes. No geral, os serviços públicos de saúde promoveram açôes educativas e divulgaram a oferta de exames de rastreamento, dando visibilidade institucional a esse nível de atenção. $O$ percurso da mulher na rede assistencial e os desafios para assegurar investigação diagnóstica e tratamento oportunos, numa visão crítica das necessidades nesse processo, foi, porém, uma abordagem bem minoritária. Como chamam a atenção Gutiérrez e Almeida (2017), ainda são grandes as desigualdades no acesso das mulheres ao diagnóstico e tratamento especializados, sendo necessária, em sua visão, a mobilizaçáo do Outubro Rosa para ampliar e qualificar o rastreamento, bem como garantir o seguimento dos casos detectados.

De fato, pouco útil será mobilizar as mulheres para a detecção precoce do câncer de mama se, após identificada uma suspeita, não se puder assegurar a elas o acesso a serviços de saúde para investigar e tratar a doença em tempo oportuno. Por sua popularidade, o Outubro Rosa tem uma força potencial para pressionar por melhorias no direito à saúde das mulheres. As vozes das pacientes e de representantes de ONGs são estratégicas nesse sentido, mas apareceram em pouco mais de $20 \%$ das matérias aqui analisadas, e o foco predominante foi o apoio social às pacientes. Políticos, de várias esferas, foram também presentes, porém na maioria das vezes em celebraçôes locais de mobilização das mulheres para a detecção precoce, mais do que fortalecendo criticamente o debate sobre o acesso ao cuidado integral à saúde. 
Cabe pontuar que a mobilização da mulher para fazer mamografia ou identificar precocemente um sinal ou sintoma suspeito de câncer de mama pode estar, em conjunto com a expansão do acesso a exames de rastreamento verificada na última década, resultando em certa antecipação do diagnóstico de câncer de mama (INCA, 2019). A inferência sobre o papel de campanhas como o Outubro Rosa nesse cenário é, porém, de difícil verificação no âmbito do estudo aqui proposto.

Visôes mais positivas ou mais céticas acerca do movimento Outubro Rosa convivem hoje e pontuam o seu papel para o avanço do controle do câncer de mama. Controlar riscos inerentes ao marketing e buscar informação ampla e de qualidade é um ponto comumente ressaltado (PUIGPINÓS-RIERA; SERRAL, 2017; CARDOSO-FILHO; SANTOS; SHINZATO, 2015). No que tange à detecção precoce, e especialmente ao rastreamento, esse é um caminho onde há muito ainda a ser trilhado.

O estudo não abrangeu todas as ações da mídia no mês de outubro, pois se limitou a uma sub-base de matérias, dentre outras disponíveis na base de dados consultada, tais como TV, rádio e vídeos. Ainda assim, o mapeamento revelado foi extenso e original e permitiu conhecer lacunas de informação que, provavelmente, são também encontradas em outros veículos.

\section{Considerações finais}

O presente estudo avaliou como a informação sobre a detecção precoce do câncer de mama foi veiculada pela mídia brasileira durante uma edição recente da campanha do Outubro Rosa. Diferentes recomendaçóes sobre faixa etária e periodicidade da mamografia são divulgadas durante essa campanha, com certo predomínio da recomendação das sociedades médicas, além de muitas recomendaçóes incompletas. Poucas matérias informaram sobre a coexistência de recomendaçôes diferentes para o rastreamento do câncer de mama, falhando em trazer o contraditório, e muito poucas trouxeram a questão dos riscos e benefícios envolvidos. Em consequência, foi praticamente ausente a temática da decisáo compartilhada.

$\mathrm{O}$ apelo às mulheres para que façam mamografia de rastreamento não pode ser dissociado da necessidade de garantir a elas informaçáo de qualidade, que apresente as incertezas nesse campo e valorize sua autonomia na tomada de decisão. As lacunas de informação indicam o quão distante está ainda o Brasil de assegurar a 
questấo ética da escolha informada no rastreamento do câncer de mama. Médicos especialistas, mastologistas e oncologistas, que predominaram como porta-vozes, devem também fazer sua parte nesse esforço que, no sistema público, envolve mais diretamente os médicos generalistas e demais profissionais da atenção primária.

Mensagens importantes como o reconhecimento das alteraçóes suspeitas de câncer de mama e a prevenção primária estão presentes no Outubro Rosa, mas poderiam ser ainda mais divulgadas. Valorizar o diagnóstico precoce e a prevenção primária, e não apenas o rastreio mamográfico, são dimensôes primordiais para o enfrentamento da doença no cenário de envelhecimento populacional brasileiro e do esperado aumento da incidência de câncer.

Além das lacunas de informação, observou-se também que muitas afirmações incorretas são propagadas pela mídia numa campanha de esclarecimento, o que traz um alerta sobre a necessidade de qualificação de parte dos veículos de imprensa. Essa observação é válida também para os profissionais de saúde que são fonte de informações, especialmente quando assumem o papel de porta-vozes em uma campanha.

Espera-se que os dados aqui apresentados contribuam para a reflexão sobre alcance e limites do papel da mídia na mobilização do Outubro Rosa na sociedade brasileira, considerando a maior complexidade hoje envolvida no propósito de conscientização sobre a doença. ${ }^{1}$

\section{Referências}

ABIGHANNAM, N; CHILEK, L. A.; KOH, H. Three Pink Decades: Breast Cancer Coverage in Magazine Advertisements. Health Communication, v. 33, n. 4, p. 462-468, 2017.

ABREU, M. M. DE et al. Apoios de decisão: instrumento de auxílio à medicina baseada em preferências. Uma revisão conceitual. Revista Brasileira de Reumatologia, v. 46, n. 4, ago. 2006.

ABREU, M. M. et al. Shared decision making in Brazil: history and current discussion. Zeitschrift für Evidenz, Fortbildung und Qualität im Gesundheitswesen, v. 105, n. 4, p. 240-244, jan. 2011.

ANDREZZO, H. F. DE A. O desafio do direito à autonomia: uma experiência de Plano de Parto no SUS. São Paulo: Universidade de São Paulo. Faculdade de Saúde Pública, 2016.

AUSTRALIAN GOVERNMENT DEPARTMENT OF HEALTH. BreastScreening and You. Information about mammography screening. AUSTRALIAN: BreastScreen, s/d. Disponível em: http://www.cancerscreening.gov.au/internet/screening/publishing.nsf/Content/194B6B D076D4A6F9CA257D71007BF9F5/\$File/Breastscreen_Brochure_March_WEB.pdf Acesso em 07 fev. 2019. 
BARDIN, L. Análise de conteúdo. São Paulo: Edições 70; 2011.

BRASIL. Secretaria de Atenção à Saúde. Departamento de Atenção Básica. Caderno de Atenção Básica. Rastreamento. v. 29. Brasília: Ministério da Saúde; 2010.

BRASIL. Ministério da Saúde. Secretaria de Vigilância à Saúde. Departamento de Análise de Situação de Saúde. Plano de Açóes Estratégicas para o enfrentamento das doenças crônicas não transmissiveis (DCNT) no Brasil. Brasília: Ministério da Saúde; 2011.

CARDOSO-FILHO, C.; SANTOS, C. C.; SHINZATO, J. Y. Outubro Rosa: o que há por trás desta iniciativa? Rev Soc Bras Clin Med., v. 13, n. 4, p. 231-232, 2015.

EBELL, M. H.; THAY, T. N; ROYALTY, K. J. Cancer screening recommendations: na international comparison of high income countries. Public Health Reviews, London, v. 39, n. 7, 2018.

ELWYN, G. et al. A three-talk model for shared decision making: multistage consultation process. BMJ (Clinical research ed.), v. 359, p. j4891, 2017.

EMERICH T. B. et al. Necessidades de saúde e direito à comunicação em tempos de Midiatização. RECIIS - Rev Eletron Comun Inf Inov Saúde, Rio de Janeiro, v. 10, n. 4, 2016.

GIGERENZER G. Reckoning with risk: learning to live with uncertainty. London: Penguin Books; 2002.

GOUVERNEMENT DU QUÉBEC. Taking part in Quebec breast cancer screening program: it's your decision. Québec: La Direction des communications du ministère de la Santé et des Services Sociaux, 2016. Disponível em www.msss.gouv.qc.ca Acesso em: 07 fev. 2019.

GUtierréZ, M. G. R.; AlmeidA, A. M. Outubro Rosa. Editorial. Acta Paulista de Enfermagem, São Paulo, v. 30, n. 5, 2017.

INSTITUTO NACIONAL DE CÂNCER JOSÉ ALENCAR GOMES DA SILVA. Estimativa 2018. Incidência do Câncer no Brasil. Rio de Janeiro: INCA, 2017.

INSTITUTO NACIONAL DE CÂNCER JOSÉ ALENCAR GOMES DA SILVA. Atlas da Mortalidade. Disponível em: http://mortalidade.inca.gov.br/Mortalidade Acesso em: 11 set. 2020.

INSTITUTO NACIONAL DE CÂNCER JOSÉ ALENCAR GOMES DA SILVA; MIGOWSKI-ROCHA-dos-SANTOS, A.; KNEIPP-DIAS, M. B. (Org.). Diretrizes para deteç̧áo precoce do câncer de mama no Brasil. Rio de Janeiro: INCA, 2015. Disponível em: https:/www.inca.gov.br/sites/ufu.sti.inca.local/files//media/document//diretrizes_deteccao_ precoce_cancer_mama_brasil.pdf. Acesso em: 22 mar. 2019.

LOWENSTEIN, L. M. et al. Active surveillance for prostate and thyroid cancers: evolution in clinical paradigms and lessons learned. Nature Reviews Clinical Oncology, 9 nov. 2018. 
McCREADY, T; LITTLEWOOD, D.; JENKINSON, J. Breast self-examination and breast awareness: a literature review. Journal of Clinical Nursing, Oxford, v.14, p. 570-578, 2005.

MARTINS, M. A. O check up do check up. Editorial. Rev Assoc Med Bras, v. 51, n. 3, p.12132, 2005.

MERCHANT, F. M.; DICKERT, N. W.; HOWARD, D. H. Mandatory Shared Decision Making by the Centers for Medicare \& Medicaid Services for Cardiovascular Procedures and Other Tests. JAMA, v. 320, n. 7, p. 641-642, 21 ago. 2018.

MIGOWSKI, A. et al. Diretrizes para detecção precoce do câncer de mama no Brasil. II - Novas recomendações nacionais, principais evidências e controvérsias. Cadernos de Saúde Pública, Rio de Janeiro, v. 34, n. 6, e00074817, 2018 a.

MIGOWSKI, A. et al. Diretrizes para detecção precoce do câncer de mama no Brasil. III - Desafios à implementação. Cadernos de Saúde Pública, Rio de Janeiro, v. 34, n. 6, e00046317, 2018b.

MIGOWSKI, A. et al. Diretrizes para detecçâo precoce do câncer de mama no Brasil. I - Métodos de elaboração. Cadernos de Saúde Pública, Rio de Janeiro, v. 34, n. 6, e00116317, 2018c.

NELSON, H. D. et al. Effectiveness of breast cancer screening: systematic review and metaanalysis to update the 2009 U.S. Preventive Services Task Force Recommendation. Ann Intern Med, v. 164, p. 244-55, 2016.

NATIONAL HEALTH SYSTEM. NHS Breast Screening Hekping you decide. United Kington: NHS / Cancer Research, 2018. Disponível em: http://www.uhs.nhs.uk/Media/SUHTInternet/ Services/BreastImagingUnit/NHS-Breast-Screening---helping-you-decide.pdf Acesso em: 07 fev. 2019.

PINK Ribbon Inc: capitalizing on hope. Direção: Léa Pool. Produção: Ravida Din. Canadá: National Film Board of Canada, 2011. 1 videocassete (97 min).

PUIGPINÓS-RIERA, R.; SERRAL, G. A favor del lazo rosa y la prevención del cáncer de mama: diferentes puntos de vista. Gac Sanit., v. 31, n. 3, p. 253-254, 2017.

ROCHA, E. M. et al. The power of a shared decision-making approach in the field of ophthalmology: will what is written in the medical and lay press work for my patient? Arquivos Brasileiros de Oftalmologia, v. 80, n. 4, 2017.

RODRIGUES, T. B. et al. Sobrerrastreio mamográfico: avaliação a partir de bases identificadas do Sistema de Informação do Câncer de Mama (SISMAMA). Cadernos de Saúde Pública, Rio de Janeiro, v. 35, n. 1, e00049718, 2019.

SILVA, A. L. Manifesto de Curitiba: pela Prevenção Quaternária e por uma Medicina sem conflitos de interesse. Revista Brasileira de Medicina de Família e Comunidade. Rio de Janeiro, v. 9, n. 33, p. 371-374, 2014. 
SIMEÃO, S. S. S. et al. A difusão do câncer de mama em mulheres na mídia impressa. Psicologia e Saber Social, Rio de Janeiro, v. 5, n. 2, p. 142-155, 2016.

STEPHAN, L. S. et al. Anticoagulação Oral na Fibrilação Atrial: Desenvolvimento e Avaliação de um Aplicativo de Saúde Móvel para Suporte à Decisão Compartilhada. Arquivos Brasileiros de Cardiologia, v. 110, n. 1, p. 7-15, jan. 2018.

URBAN, L. A. B. D. et al. Recomendaçóes do Colégio Brasileiro de Radiologia e Diagnóstico por Imagem, da Sociedade Brasileira de Mastologia e da Federação Brasileira das Associaçóes de Ginecologia e Obstetrícia para o rastreamento do câncer de mama. Radiologia Brasileira, São Paulo, v. 50, n. 4, p. 244-249, 2017.

VASCONCELLOS-SILVA, P. R. et al. As novas tecnologias da informação e o consumismo em saúde. Cad. Saúde Pública, Rio de Janeiro, v. 26, n. 8, p. 1473-1482, 2010.

VASCONCELLOS-SILVA, P. R.; SORMUNEM, T.; CRAFTMAN, A. G. Evolution of acess to information on breast cancer and screening on the Brazilian National Cancer Institute website: na exploratory study. Ciência e Saúde Coletiva, Rio de Janeiro, v. 23, n. 4, p.1303-1312, 2018.

VIEIRA, S. C. Câncer de mama: Consenso da Sociedade Brasileira de Mastologia - Regional Piauí. Teresina: EDUFPI, 2017.

WORLD CANCER RESEARCH FUND. AMERICAN INSTITUTE FOR CANCER RESEARCH. Diet, nutrition, physical activity and breast cancer. Washington DC: AICR, 2017. Revisado em 2018. Disponível em: <https://www.wcrf.org/sites/default/files/Breastcancer-report.pdf> Acesso em: 27 fev. 2019.

WORLD HEALTH ORGANIZATION. WHO position paper on mammography screening. Geneva, 2014. Disponível em https://apps.who.int/iris/bitstream/ handle/10665/137339/9789241507936_eng.pdf;jsessionid=A12BC5A174E204F64FFBCBD3 71620870?sequence=1 Acesso em: 27 mar. 2019.

YAMAMOTO, S. T. Desencontros entre direitos e desejo da mulher e a decisão da equipe médica na prática da esterilização cirúrgica. São Paulo: Universidade de São Paulo. Faculdade de Saúde Pública, 2017.

\section{Nota}

${ }^{1}$ M. de Assis e R. O. M. dos Santos participaram de todas as etapas da pesquisa e da redação do artigo. A. Migowski participou da concepção, revisão metodológica e revisão do artigo. 


\section{Abstract}

\section{Breast cancer early detection in the Brazilian media during the Breast Awareness Month} This study aimed to analyze how the information on early detection of breast cancer is presented in the Brazilian media during the Breast Awareness Month. News published in October 2017 on the Internet were analyzed. The search retrieved 1,011 News, which resulted in the selection of 345 eligible News. The main spokespersons were the mastologists (17.1\%) and the patients (13.0\%) and the predominant institutional links were public (36\%) and private (34\%). Early detection was the second most frequent theme (55\%) and mammographic screening was addressed in $79.1 \%$ of them. The recommendation to start mammographic screening at the age of $40(34.3 \%)$ predominated when compared to 50 years or more $(29.3 \%)$. The government recommendation for biennial screening from 50 to 69 years was cited in $17.5 \%$ of the News. Only $3.3 \%$ cited screening harms and $1.5 \%$ the shared decision making. Suspected signs and symptoms of breast cancer were reported in $30 \%$ of the News and only $11.8 \%$ listed all the main ones. The lack of information and the presence of inadequate or incomplete recommendations regarding age and screening interval show the limit of the campaign in disseminating information based on the best evidence.

> Keywords: breast neoplasms; early detection of cancer; mass screening; mammography; health communications. 